\title{
Comparison of Single Vehicle Noise Emission Models in Simulations and in a Real Case Study by means of Quantitative Indicators
}

\author{
Gabriella Graziuso $^{1}$, Simona Mancini ${ }^{2}$ and Claudio Guarnaccia ${ }^{1, *}$ \\ ${ }^{1}$ Department of Civil Engineering, University of Salerno, \\ via Giovanni Paolo II 132, 84084, Fisciano, SA, Italy \\ ${ }^{2}$ Department of Information and Electric Engineering and Applied Mathematics, University of \\ Salerno, via Giovanni Paolo II 132, 84084, Fisciano, SA, Italy
}

Received: May 15, 2020. Revised: July 27, 2020. 2nd Revised: September 16, 2020. Accepted: October 19, 2020. Published: October 27, 2020.

\begin{abstract}
Road traffic has a significant influence on the environmental noise pollution, producing harmful effects on human health and collective well-being. Since noise measurements cannot be performed everywhere, or even in a large number of sites, because of high costs and time consumption, traffic noise predictions are necessary for noise abatement and control. Consequently, many scientific models have been developed in recent years focusing on this aspect with the definition of source emission and sound propagation empirical formulations exclusively. This paper focuses on some Emission Models, describing the comparison of the results, in terms of sound power level emitted by a single vehicle, obtained with their application to different simulations of driving conditions and to two real study cases. After a preliminary comparison between the models, the evaluation of two indicators, namely the average and total source power level, will be discussed in relation to different conditions of the vehicle kinematics. The application of these two indicators to real study cases will demonstrate their validity. It will be shown that this procedure can become a tool for supporting people route choices according to a less impact of environmental noise. This will open the way to new scenarios of eco-routing, by means of implementation of models prediction in dedicated software platforms and/or in car navigation systems.
\end{abstract}

Keywords - Noise Predictive Models, Road Traffic Noise, Simulation, Sound Power Level.

\section{INTRODUCTION}

CURRENTLY, significant cases of heart disease and premature deaths can be related to long term exposure to noise pollution. According to the European Environment Agency [1], the $20 \%$ of the EU's population lives in areas where noise levels are considered harmful to human health and well-being. Specifically, road traffic noise is the most significant source of environmental noise pollution in cities with more than 110 million people affected by higher average noise levels than the standard threshold (55 dBA) daily. Moreover, an increase in numbers is expected in the near future because of urban growth and increased mobility demands.

In order to reduce noise pollution, the Environmental Noise Directive 2002/49/EC [2] enforces EU member States to start a process of its management and containment which involves the knowledge of its degree and the number of exposed people, the preparation of action plans, the information sharing, the public involvement and participation. In particular, the environmental noise impact can be evaluated through the analysis of the data deriving from a measurement campaign, with the use of accurate instruments and in compliance with the technical regulations, or the use of a software simulation. Even though field measurements are more accurate than software predictions, it is very difficult to plan and implement a large scale measurement campaign. As a consequence, from the middle of the last century, several models to predict environmental noise have been developed, focusing in particular to noise produced by transportation. Road traffic, in particular, is commonly assumed to be the 
principal source in urban areas [3]-[11], that affects noise levels at the buildings' facades [12]. However, the Road Traffic Noise predictive Models (RTNMs) need a very precise mathematical modelling of the sources, the sound propagation law and the surrounding environment. Specifically, the estimation of the noise emissions generated by road traffic requires the knowledge of traffic flow, both of light and heavy vehicles, the vehicle kinematics, vehicle engines, exhaust systems, tyre-pavement interaction and aerodynamic friction [8], [9], and the environmental features, i.e. characteristics of the road surface, weather and distance between carriage and receivers [10], [13].

In this paper, the authors present the main Noise Emission Models (NEMs), generally used to estimate the source power level of a single vehicle and, by aggregation of more vehicles, of a traffic flow. These NEMs are compared each other through the definition of two indicators, namely the average and total sound power level (section 2). Specifically, the analysis of the models will be based on the input of speed profiles related both to simulations and field data collected with a GPS speedometer application on urban and suburban routes (section 3). The results will show that the two indicators are useful tools to be used in "eco-routing", i.e. in routes choices according to the environmental consequences. The driver, in fact, when choosing the destination and the possible route, could be informed on the noise emission predicted in the different routes, allowing a choice based on environmental sensibility. The idea of including noise assessment in eco-routing is an innovative and new scenario, since it usually includes just fuel consumption and air pollutants emissions.

\section{MATERIAL AND METHODS}

In order to predict the road traffic noise, several RTNMs were developed in different countries. Some of the most popular ones are listed in Table 1. Although the characterization of source in terms of rolling and propulsion noise in conjunction with advanced numerical methods for sound propagation has significantly reduced the uncertainty in traffic noise predictions, yet sometimes the approach followed is quite complex and requires specialized mathematical skills for predictions [14].

The different models are based on both a statistical [3]-[6], [8], [15] and a dynamical approach [10], [13], [16] through the evaluation of the vehicle kinematics, namely position, speed and acceleration, which can give a reliable description of real noise emission. In particular, speed profiles are extremely important, in order to properly assess the noise emitted in different regimes. Specifically, the first approaches were statistical and based on the implementation of models whose parameters were obtained by a regression of experimental data, obtained through field measurements and noise data collection, for a representative sample of vehicles [17]. These models were based on constant speed simulations, as the predicted levels were expressed as functions of speed, and with zero acceleration [8]. Later models predicted the equivalent continuous level $\left(\mathrm{L}_{\mathrm{eq}}\right)$ for traffic over a chosen period and the maximum A-weighted sound level $\mathrm{L}_{\mathrm{Amax}}$ is measured at a given reference distance $d$ from the road [10]. These maximum levels can be converted into the sound power level $\mathrm{L}_{\mathrm{w}}$ emitted by the vehicle, considering an omnidirectional source and the energy of the ground [17]. Still earlier models considered different flow conditions (i.e. interrupted and varying/pulsed) and classified road vehicles into several categories [18], [19].

Table 1. RTN models and their main applications.

\begin{tabular}{|c|c|c|}
\hline $\begin{array}{l}\text { Road Traffic } \\
\text { Noise Model } \\
\end{array}$ & Country & Main applications \\
\hline $\begin{array}{l}\text { ASJ RTN- } \\
2008\end{array}$ & Japan & $\begin{array}{l}\text { Highway. Constant speed. } \\
\text { Different traffic } \\
\text { conditions }\end{array}$ \\
\hline Cnossos & Europe & $\begin{array}{l}\text { Road traffic and other } \\
\text { sources }\end{array}$ \\
\hline CoRTN & $\begin{array}{l}\text { UK, Australia, } \\
\text { Hong Kong, } \\
\text { New Zealand }\end{array}$ & $\begin{array}{l}\text { Highway. Single traffic } \\
\text { stream }\end{array}$ \\
\hline FHWA & $\begin{array}{l}\text { USA, Canada, } \\
\text { Japan, Mexico }\end{array}$ & Highway, road networks \\
\hline Harmonoise & Europe & Road and railway traffic \\
\hline Imagine & Europe & Road and railway traffic \\
\hline MITHRA & $\begin{array}{l}\text { France, } \\
\text { Belgium }\end{array}$ & $\begin{array}{l}\text { Highways and railways. } \\
\text { Simple streams }\end{array}$ \\
\hline $\begin{array}{l}\text { NMPB Routes } \\
-2008\end{array}$ & France & $\begin{array}{l}\text { Highway and road } \\
\text { networks }\end{array}$ \\
\hline Nord 2000 & $\begin{array}{l}\text { Norway, } \\
\text { Denmark, } \\
\text { Sweden, } \\
\text { Finland }\end{array}$ & $\begin{array}{l}\text { Source model for road } \\
\text { and rail traffic }\end{array}$ \\
\hline RLS 90 & Germany & $\begin{array}{l}\text { Highways. Car parks. } \\
\text { Simple streams only }\end{array}$ \\
\hline SonRoad & Switzerland & $\begin{array}{l}\text { Highway and road } \\
\text { networks }\end{array}$ \\
\hline STL-86 & Switzerland & $\begin{array}{l}\text { Highways and trams, light } \\
\text { rail. Simple streams }\end{array}$ \\
\hline
\end{tabular}

A. Noise emission models for the evaluation of the sound power level

Based on literature, the present study will focus on light vehicles noise emission estimated according to five models, that are Lelong [20], SonRoad [21], NMPB Routes [22], [23], ASJ-RTN (both for steady and non-steady conditions) [24] and Cnossos [25]. These models adopt the usual statistical structure, made of a function with coefficients estimated by regression on a calibration dataset. Of course, this makes the models "site dependent" from a general point of view [5]. Anyway, the specific features of each model are not always influent in the applications, since, as it will be shown later in this paper, the patterns of the results are very similar when applied to simulated or real case study data. The general 
formulas and parameters are shortly resumed in this section.

Lelong Model (used in [15]) is based on the noise prediction of traffic using the amount of acoustic energy sent to the receiver by the single vehicle and is linked to its speed through a mathematical relationship. In particular, it considers two speed regimes: cruising/decelerating and accelerating flows. The model considers a constant $\mathrm{L}_{\mathrm{w}}=82 \mathrm{dBA}$ for $\mathrm{v} \leq 11.5 \mathrm{~km} / \mathrm{h}$, for the first regime, and a constant $\mathrm{L}_{\mathrm{w}}=90.5 \mathrm{dBA}$ for $\mathrm{v} \leq 25$ $\mathrm{km} / \mathrm{h}$, for the accelerating flow. For other speed values, the relation is:

$$
L_{W}(v)=\alpha_{L}+\beta_{L} \log v
$$

$$
\text { with } \alpha_{\mathrm{L}}=53.6 \pm 0.3 \mathrm{dBA} \text { e } \beta_{\mathrm{L}}=26.8 \pm 0.2 \mathrm{dBA} \text {. }
$$

The Swiss SonRoad Model [21] defines the noise produced by a single vehicle according to its speed and type, as well as the uphill grade $(\Delta s)$ and the surface type of the road $\left(\Delta_{\mathrm{BG}}\right)$. Latest source models use separate formulas for passenger cars and trucks. It estimates the A-weighted maximum pass-by level of a single vehicle at a distance of $7.5 \mathrm{~m}$ and a height of $1.2 \mathrm{~m}$ above the ground, for 3500 pass-byes measurements. The procedure orders the pass-by events according to the vehicle speed, defining speed classes of $4 \mathrm{~km} / \mathrm{h}$. Within each speed class, the average energy value can be calculated and, finally, the A-weighted power level can be evaluated:

$$
\begin{gathered}
\mathrm{L}_{\mathrm{W}, \mathrm{A}, \text { passenger }}=28.5+ \\
+\log \left(10^{0.1(7.3+35 \log \mathrm{v})}+10^{0.1\left(60.5+10 \log \left(1+(\mathrm{v} / 44)^{3.5}\right)+\Delta_{s}\right)}\right)+ \\
+\Delta_{\mathrm{BG}} .
\end{gathered}
$$

The emission values of the NMPB Routes 2008 Model [22], [23] are expressed through the power contributions, as a function of the traffic speed, type of traffic flow, the road platform surface state and categories and the road gradient. In this model, the analysis of the sound power level is also characterized of two contributions, i.e. rolling and power unit noise. These components are determined through experimental results, obtained at $7.5 \mathrm{~m}$ horizontal distance from the road and $1.2 \mathrm{~m}$ height above the ground. The A-weighted pass-by maximum level can be calculated with the following expression:

$$
L_{A, \max }(v)=L_{p}+L_{r}=10 \log \left(10^{0.1 L_{p}}+\left(10^{0.1 L_{r}}\right)\right)
$$

$L_{p}$ and $L_{r}$ represent respectively the power unit noise and rolling power levels. The power unit noise level is defined in relation to the speed and according to the acceleration, deceleration or steady state, while the rolling component depends on the different categories of vehicles (light or heavy) and for three different road surface categories. Then, the sound power level for a single vehicle is expressed by:

$$
L_{w}(v)=L_{A, \max }(v)+\log \frac{d}{d_{0}}+10 \log 2 \pi
$$

with $\frac{d}{d_{0}}=\sqrt{7.5^{2}+1.2^{2}}$.

ASJ-RTN 2008 Model [24] is the latest version used in Japan for the study the sound propagation and the description of the source emission. The model divides vehicles within three (light and heavy vehicles, and motorcycles) or four categories (passenger cars, small-sized vehicles, medium-sized vehicles and heavy-sized vehicles, and motorcycles) and introduces methods of calculation of the A-weighted sound power at intersections and in different driving conditions, with correction for different type of asphalt.

The sound power level emitted by a car is defined as:

$$
L_{W, A}=a+b \log (V)+C
$$

where $V$ is the vehicle speed in $\mathrm{km} / \mathrm{h}$. The coefficients $a$ and $b$ vary according to the steady and non-steady traffic conditions. Moreover, $C$ is the correction term that considers, among others, the draining asphalt and the gradient of roads, the directivity of sound radiation and other specific factors. For a passenger car in a traffic flow section the parameters are:

- $\quad a=46.4 \mathrm{dBA}$ and $b=30$, in steady traffic conditions;

- $\quad a=82.0 \mathrm{dBA}$ and $b=10$, in non-steady conditions.

With regards to the non-steady conditions of acceleration and deceleration, the model analyses three different cases for the calculation of the above-mentioned parameters: proximity to an expressway tollgate, a junction or a signalized intersection.

The European Commission developed a methodological framework (Common Noise Assessment Methods CNOSSOS) for noise emissions deriving from road and rail traffic, aircraft and industrial activities. In relation to the road traffic noise emissions, the vehicles are divided into four categories (light, medium, heavy and two-wheeled vehicles) in the CNOSSOS Model [25]. A fifth category is reserved to vehicles that will be introduced in the future, as they may require additional considerations in order to properly evaluate the noise. This category would include, for example, hybrid vehicles or with a different type of propulsion.

Analysing a single vehicle, the sound power level considers mainly the source category and its speed, with the necessary corrections due to the environmental effects and the specific ones. For light, medium and heavy vehicles, in relation to certain weather and traffic conditions, the overall sound power level is:

$$
L_{W, i, m}\left(v_{m}\right)=10 \log \left(10^{\frac{L_{W R, i, m}\left(v_{m}\right)}{10}}+10^{\frac{L_{W P, i, m}\left(v_{m}\right)}{10}}\right) .
$$

It is expressed in relation of the two components of rolling and propulsion.

For the rolling noise, the power level can be expressed 
through the following logarithmic formula:

$$
L_{W R, i, m}\left(v_{m}\right)=A_{R, i, m}+B_{R, i, m} \cdot \log \left(\frac{v_{m}}{v_{r e f}}\right)+\Delta L_{W R, i, m}\left(v_{m}\right)
$$

where $v_{\text {ref }}$ is the reference speed of $70 \mathrm{~km} / \mathrm{h}$ and the coefficients A and B depend on the category of vehicle and are given in octave bands, from $63 \mathrm{~Hz}$ to $8 \mathrm{kHz} . \Delta L_{W R, i, m}\left(v_{m}\right)$ is calculated as the sum of some corrective coefficients related to the road surface, the type of tyre, the presence of intersections or roundabouts, and the environmental temperature.

For the propulsion noise, the sound power level is expressed by the following linear formula:

$$
L_{W P, i, m}=A_{P, i, m}+B_{P, i, m} \cdot\left(\frac{v_{m-} v_{r e f}}{v_{r e f}}\right)+\Delta L_{W P, i, m}\left(v_{m}\right)
$$

where the coefficients are evaluated similarly to the previous rolling noise. Also $\Delta L_{W P, i, m}\left(v_{m}\right)$ depends on the road surface and gradient, the type of vehicle and its acceleration/deceleration.

In accordance with their meaning, Figure 1 shows that that the propulsion sound power level is higher at low speeds, while the rolling power level is higher at high speeds.

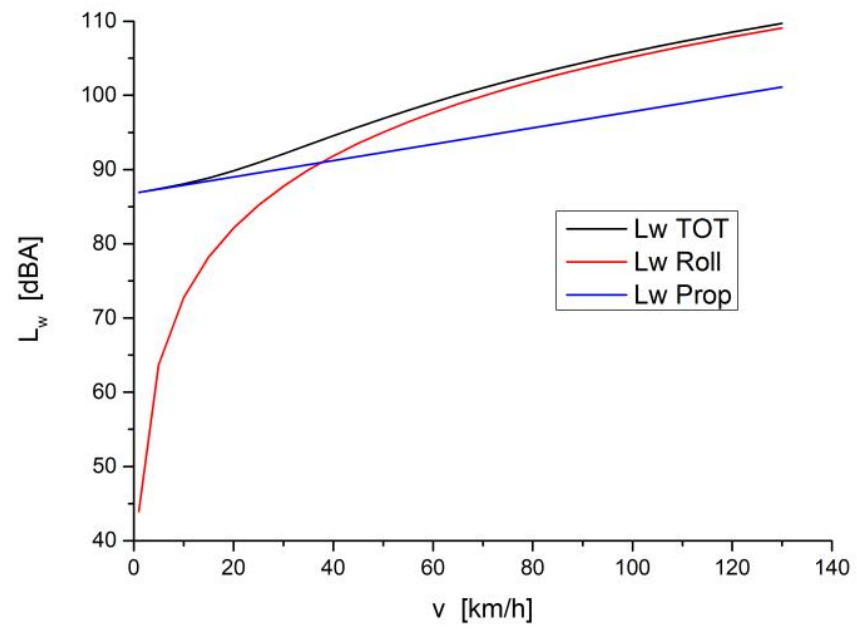

Figure 1. Cnossos model: A-weighted sound power level for a single vehicle.

\section{B. Noise emission models comparison as a function of the vehicle speed}

It is difficult to define the pros and cons of the different approaches, above all because these models have been validated in their respective countries they were ideated. Also, the idea of an ideal model proposed by Steele [8] has been already overcome by more recent models, because they are more exhaustive than the previous ones.

Basically, recent models separate the source and propagation parts, in order to allow the updating of their laws independently. Moreover, they recommend the separation of vehicle noise into propulsion and rolling components. This allows to include specific corrections, such as slope for engine noise and speed or pavement for tyre noise.

All the models generally present continuous functions in speed that include a logarithmic term. However, functions differ between the models, both in shape and coefficients. Their discrepancies are basically related to different choices of modelling and to different data used for the calibration, that means a large variation in the coefficients of each model. In some sense this is typical of statistical models, that are influenced by the site in which calibration data are collected.

The way the models take into account vehicle acceleration is very variable. For example, SonRoad model does not account for acceleration, probably because it is dedicated to suburban noise inventories, while Cnossos proposes a correction for urban driving conditions, based on the distance to the intersection [10].

Several varied terms of correction are also considered in each model, in relation to different conditions, such as: the type of vehicle and its acceleration/deceleration, the type of tyre, vehicular density and traffic flow characteristics, the presence of intersections or roundabouts, the road surface and gradient, the environmental temperature and meteorological effects.

In order to compare the above-mentioned models, Figure 2 shows the trend of the sound power level of a single vehicle as a function of speed (given in $\mathrm{km} / \mathrm{h}$ ). Such a comparison has been carried out in an ideal scenario, represented by a straight road, without changes in altitude (hence, with zero gradient), made of common asphalt (neglecting, then, the corrections due to the road surface) and without considering the possible interactions with other vehicles. Almost all the models have a similar logarithmic trend, especially at medium-high speed, when the noise generated by tire-asphalt contact and rolling is predominant. At low speeds, instead, when the propulsion noise prevails, the differences between the models are more evident.

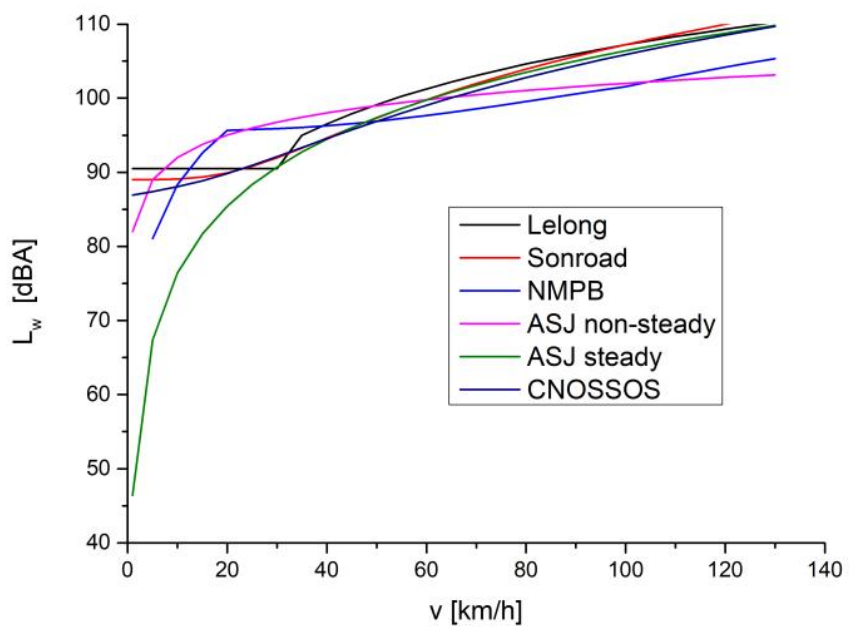

Figure 2. Comparison of models: A-weighted sound power level for a single vehicle. 


\section{Definition of indicators for models comparison}

Since the main goal of this study is the assessment of the sound power level of a single vehicle when its dynamic conditions vary, a quantitative comparison between the results deriving from the application of the above presented models can be made. Consequently, two indicators can be defined in relation to the average and the total sound power level in a certain time of observation or for a origin-destination path:

$$
\begin{gathered}
L_{w, m}=10 \log \left[\frac{1}{T} \sum_{i} 10^{L w, i} \Delta t\right] ; \\
L_{w, t o t}=10 \log \left[\sum_{i} 10^{L w, i}\right]
\end{gathered}
$$

where $L_{w, i}$ is the sound power level emitted by the vehicle in the $i$-th time interval, $\Delta t$ is the duration of the $i$-th time interval used for measurement or estimation of sound power level and $\mathrm{T}$ is the total time of observation of the phenomenon. It can be noticed that the first indicator provides an average information, estimating the mean noise emission level during a trip. On the other side, the second indicator provides a global information, estimating the overall noise emission level, summing all the "instantaneous" sound power emitted by a vehicle along a full route.

\section{SIMULATIONS RESULTS AND DISCUSSION}

In order to evaluate the differences between the abovementioned emission models according to kinematics of a single light vehicle, all the correction terms will be neglected. Therefore, it is necessary to have information about the speed and acceleration, that are usually known with a poor resolution [10]. Indeed, for example, the mean speeds are not known for every road segment and speed distributions are rarely available, especially near intersections [26], [27]. To overcome this lack of information for the present study, the five models will be applied to three simulations and two real cases in different speed conditions.

\section{A. Simulations results}

The simulations mimic a light passenger vehicle running on a straight road, with three different speed profiles. The time of simulation is fixed and corresponds to an interval of 60 seconds. According to the different speed profiles, a different length is travelled. The speed values are given in input to the NEMs described in Section II, producing the curves of source power level of the simulated vehicle as a function of time. The simulations are performed in Matlab $\subset$ and Microsoft Excel@.

The three speed distributions have the aim to simulate a free flow traffic condition (Simulation 1) on a high-speed road (e.g. highway) and two different driving conditions on an urban road: a stop\&go condition, for instance in proximity of an intersection with traffic light (Simulation 2), and a random acceleration condition, for instance in a congested road (Simulation 3).

The free flow condition in Simulation 1 aims at simulating the entering a highway from the acceleration lane. The speed profile, shown in Figure 3 in dashed red line, with a secondary axis, starts from $11.1 \mathrm{~m} / \mathrm{s}(40 \mathrm{~km} / \mathrm{h})$ and is characterized by a 10 seconds time interval of constant acceleration $\left(2 \mathrm{~m} / \mathrm{s}^{2}\right)$, simulating the vehicle entering a highway. After the 10 seconds of acceleration, the vehicle runs with constant speed till the end of the observation time. The corresponding sound power level curves for the various models are presented in Figure 3 (coloured solid lines).

The speed profile of simulation 2 (Fig. 4 in dashed red line, with a secondary axis) wants to represent a light vehicle running on a urban road, in proximity to a traffic light. The vehicle starts from zero speed and accelerates for 10 seconds, with constant acceleration equal to $1.3 \mathrm{~m} / \mathrm{s}^{2}$. After 8 seconds of steady speed, it decelerates to stop at the simulated red traffic light. The stop at the traffic light occurs at the second 22 and lasts 10 seconds. Then, the car accelerates with constant acceleration equal to $1.5 \mathrm{~m} / \mathrm{s}^{2}$, up to $15 \mathrm{~m} / \mathrm{s}(54$ $\mathrm{km} / \mathrm{h})$. The sound power levels curves are reported in Figure 4 (coloured solid lines).

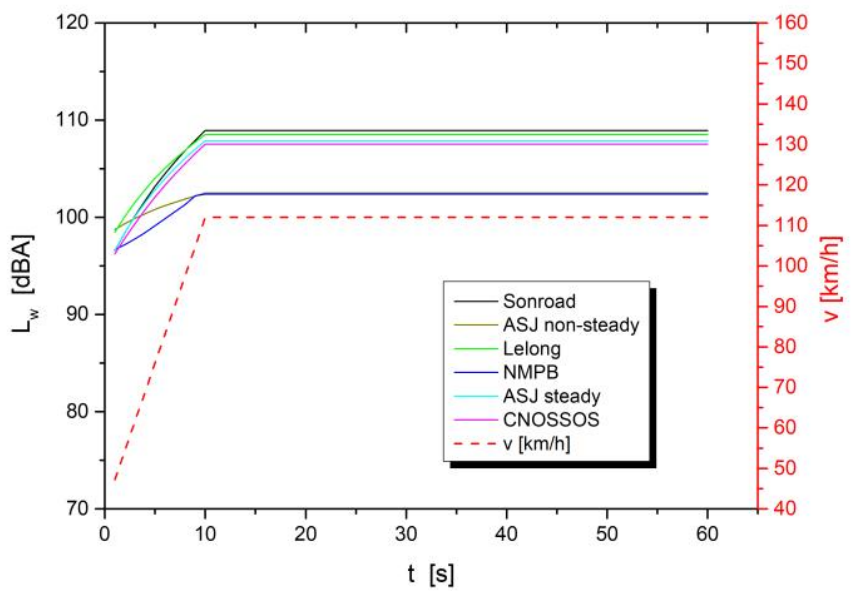

Figure 3. Simulation 1: Speed profile (in dashed red line, secondary axis) and sound power level $L_{w}$ of the different models (solid lines)

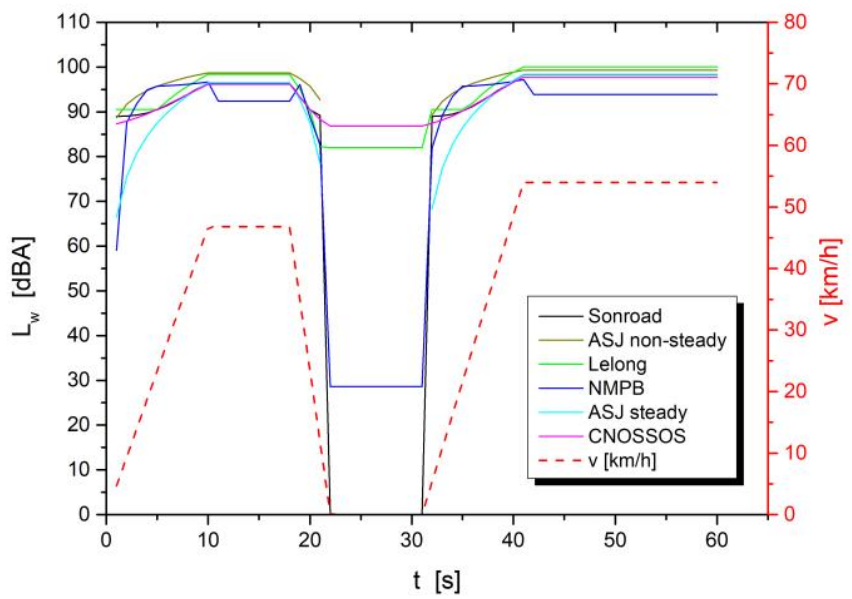

Figure 4. Simulation 2: Speed profile (in dashed red line, secondary axis) and sound power level $L_{w}$ of the different models (solid lines) 
The last simulation is related to a highly variable motion, characterized by a random acceleration instant by instant. Generally, it varies in magnitude and in positive/negative direction, except for the first 10 seconds, where the direction is only positive. Figure 5 shows the speed profile versus time (in dashed red line, with a secondary axis) and the corresponding source power level curves (coloured solid lines).

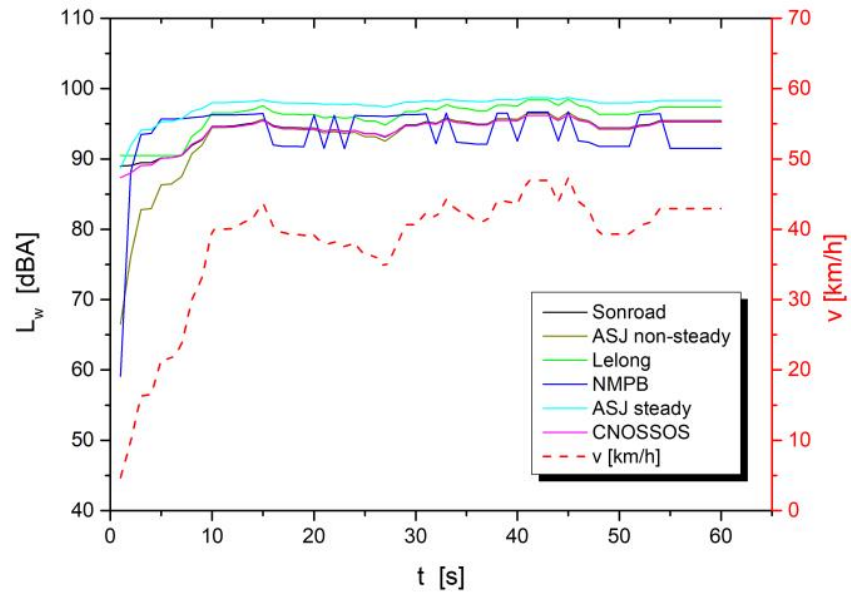

Figure 5. Simulation 3: Speed profile (in dashed red line, secondary axis) and sound power level $L_{w}$ of the different models (solid lines)

\section{B. Simulations discussions}

Simulation 1 shows that almost all the models have similar trends, except for NMPB and ASJ-RTN non-steady, which have lower values. The latter, indeed, is valid up to a speed of $80 \mathrm{~km} / \mathrm{h}$ on highways, that is reached in about 8 seconds; after this value the steady model should be considered.

As for Simulation 2, it is possible to focus on idling stopped vehicles at traffic light. For SonRoad and ASJ models, the power level turns out to be zero, because they consider only the sound emissions as a function of different speeds from zero. NMPB model, instead, considers an extremely low power level (approximately $28 \mathrm{dBA}$ ) that is almost unrealistic if considering that the emission is approximately $91 \mathrm{dBA}$ at 10 $\mathrm{km} / \mathrm{h}$. In the other ranges of time, almost all models are congruent with each other. The only exception is the NMPB that presents a sudden decrease in the level every time that the vehicle changes its motion from "uniform" to "accelerated" regime. This peculiarity is justified by the abrupt transition from steady to non-steady motion.

Simulation 3 describes common real situations of busy roads (with consequent motion disturbance of vehicles) or an aggressive driving style. This is the only simulation that presents a jerk (i.e. the acceleration derivative) different from zero, since in the previous ones it was null. In Figure 5 it is possible to note the similarity between almost all methods, which, after the first phase of acceleration, converge to values very close to each other. Only NMPB model has a fluctuation of values. Moreover, it is interesting to observe the similarity between ASJ non-steady and Cnossos, even though they are based on quite different principles.

A quantitative comparison between the three simulations can be realized with the calculation of the two indicators $\mathrm{L}_{\mathrm{w}, \mathrm{m}}$ and $\mathrm{L}_{\mathrm{w} \text {,tot }}$, presented in Section II.C. The results are presented in Table 2. As expected from the definition of the two indicators, the total sound power levels assume a higher value than the average ones, which are averaged over the total time. Moreover, the speed profile of simulation 1 produces greater power levels, both medium and total. Finally, analyzing the individual simulations with reference to the various models, they show similar results, since the differences are generally contained within a $\pm 3 \mathrm{dBA}$ range, with the exception of the NMPB model, that exhibits values usually lower than the others.

Table 2. Comparison of simulations. All values are given in dBA.

\begin{tabular}{l|cc|cc|cc}
\hline \multirow{2}{*}{ Models } & \multicolumn{2}{|c|}{ Simulation 1 } & \multicolumn{2}{c|}{ Simulation 2 } & \multicolumn{2}{c}{ Simulation 3 } \\
& $\mathrm{L}_{\mathrm{w}, \mathrm{m}}$ & $\mathrm{L}_{\mathrm{w}, \text { tot }}$ & $\mathrm{L}_{\mathrm{w}, \mathrm{m}}$ & $\mathrm{L}_{\mathrm{w}, \text { tot }}$ & $\mathrm{L}_{\mathrm{w}, \mathrm{m}}$ & $\mathrm{L}_{\mathrm{w}, \text { tot }}$ \\
\hline Lelong & 108.1 & 125.9 & 97.2 & 115.0 & 96.5 & 114.2 \\
SonRoad & 108.5 & 126.3 & 95.6 & 113.3 & 94.6 & 112.4 \\
NMPB & 102.1 & 119.8 & 93.3 & 111.1 & 94.7 & 112.4 \\
ASJ non-steady & 102.3 & 120.1 & 97.3 & 115.1 & 97.8 & 115.6 \\
ASJ steady & 107.4 & 125.2 & 95.4 & 113.2 & 94.4 & 112.1 \\
CNOSSOS & 107.1 & 124.8 & 95.1 & 112.9 & 94.4 & 112.2 \\
\hline Average & $\mathbf{1 0 5 . 9}$ & $\mathbf{1 2 3 . 7}$ & $\mathbf{9 5 . 7}$ & $\mathbf{1 1 3 . 4}$ & $\mathbf{9 5 . 4}$ & $\mathbf{1 1 3 . 2}$ \\
\hline
\end{tabular}

\section{CASE STUDY}

In order to implement the previous analysis in a real case study, the models have been applied to two different routes (Fig. 6). They concern the SS18 national road and the E841 highway, connecting the same origin and destination points: i.e. the Baronissi Campus of the University of Salerno (South Italy), and the Marco Mattiucci bridge, close to the Ghirelli theatre, in Salerno.

The routes have been covered in both directions. Thanks to their almost parallel configuration and the approximations of the correction terms of the models, it is possible to calculate the variation of the sound power level $\left(\mathrm{L}_{\mathrm{w}}\right)$ in relation only to their speed profile.

In this application, the speed data collection has been acquired with a GPS speedometer application for mobile devices (Fig. 7(a)). Using the GPS data, the app reproduces a digital speedometer that measures speed, distance, time, location, altitude and degree of elevation. The outputs are the instantaneous, average and maximum speeds. Through this application it has been possible to collect data during the travel, visualize them (Fig. 7(b)) and extract a spreadsheet of the measured elements [28]. 

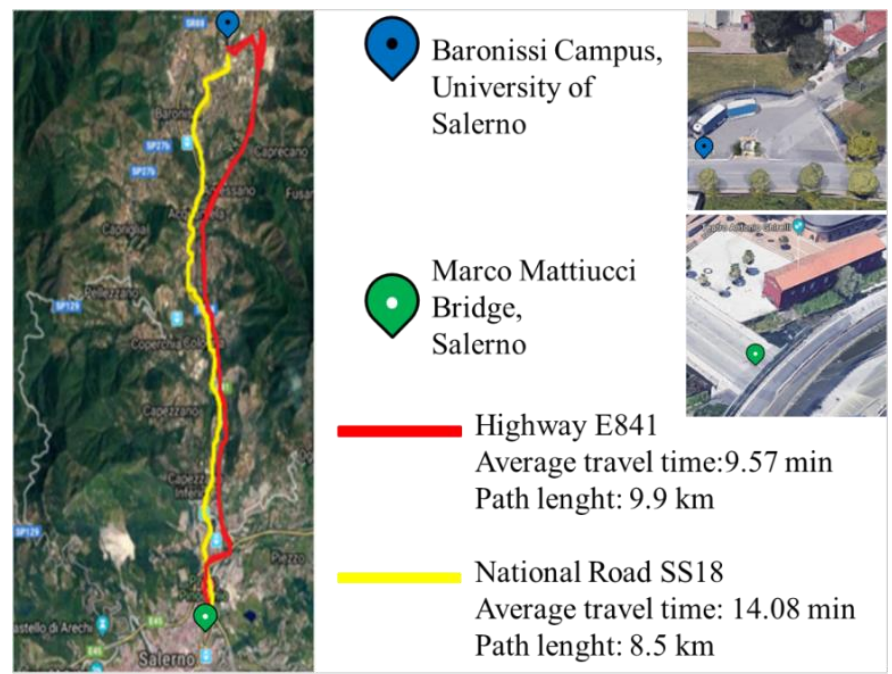

Figure 6. Case study details and routes.
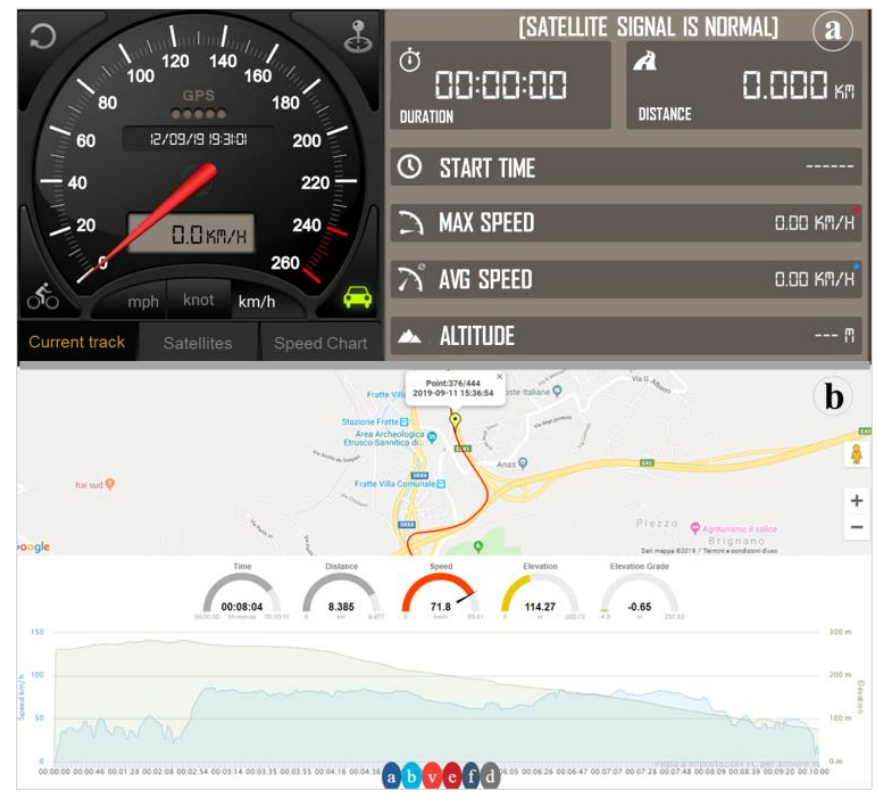

Figure 7. (a) GPS speedometer, (b) Data collection visualization.

\section{A. Highway routes}

The E841highway has emergency lanes only in a limited part of the route and the vehicle speed limit is almost everywhere fixed at $80 \mathrm{~km} / \mathrm{h}$, except for a dangerous curve in correspondence of a tunnel, where the limit is $60 \mathrm{~km} / \mathrm{h}$. The red dashed lines in Figure 8 and Figure 9 show the slope of the speed, measured with the speedometer, in function of the time for both directions. The speed trends are similar, especially in the middle sector, that corresponds to the highway section. The start and end sections, instead, are highly variable because they correspond to urban roads that connect the destinations and the highway acceleration lane and are characterized by variable traffic conditions.

The coloured solid lines in Figure 8 and Figure 9 show the sound power levels calculated with the various models in both highway routes. The functions exhibit a trend similar to the one analysed in the simulations. NMPB, indeed, tends to provide lower levels, while the other models are similar. In addition, it is possible to observe a strongly variable sound power level for the first and the last 100 seconds, and more homogeneous and higher levels in the central part of the route.

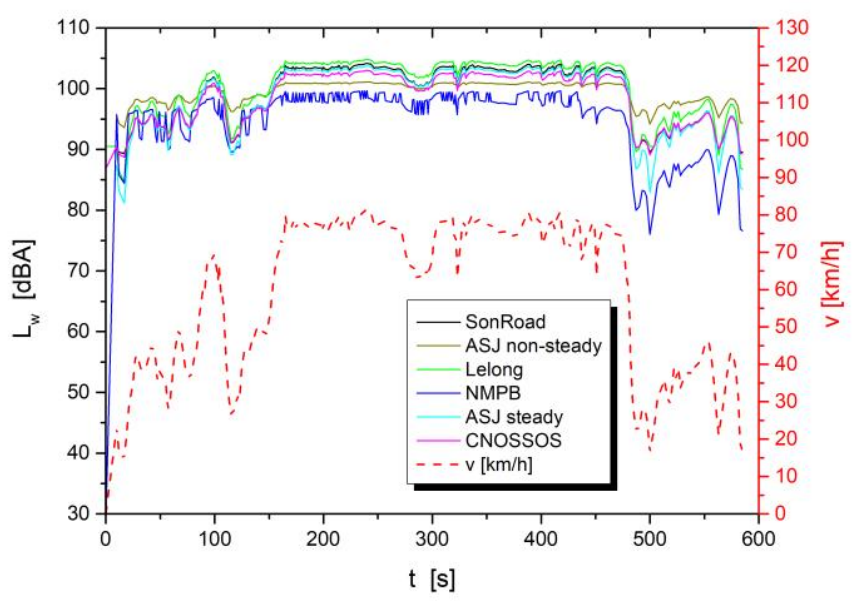

Figure 8. Highway route Salerno-Baronissi: Speed profile (in dashed red line, secondary axis) and sound power level $L_{w}$ of the different models (solid lines).

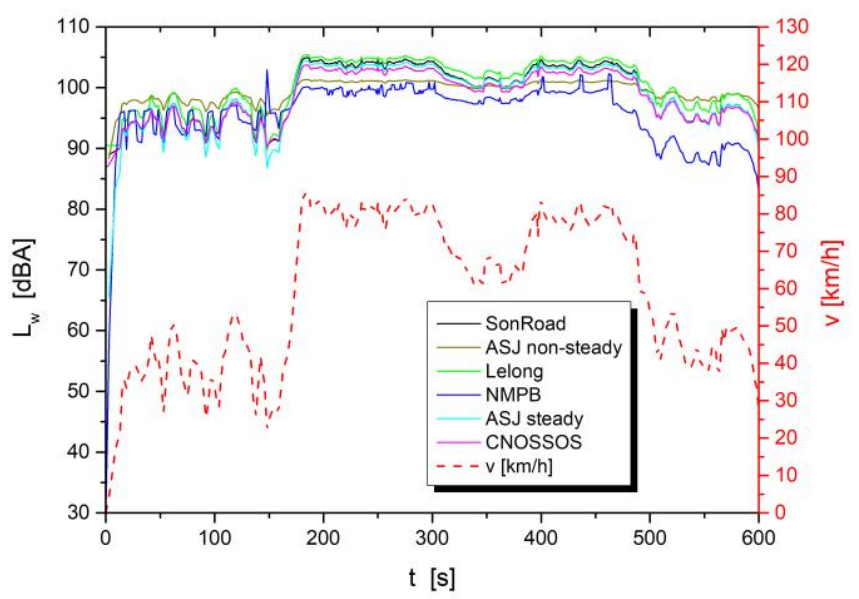

Figure 9. Highway route Baronissi-Salerno: Speed profile (in dashed red line, secondary axis) and sound power level $L_{w}$ of the different models (solid lines).

\section{B. National road routes}

The SS18 National Road, travelled in both directions, is an urban road crossing the towns of Baronissi, Pellezzano and Salerno. Mainly, it is characterized by a limited number of intersections, mostly located in correspondence of the urban centres. It has slightly higher duration than the previous one, of about 14 minutes. The speed profiles are extremely similar in both directions, with few differences in the section with the destination localized in Baronissi. The red dashed lines in Figure 10 and Figure 11 show the speed profiles, with lower values than the one measured on the highway route. However, they are also highly variable, because of the urban traffic and the presence of curves and intersections on the road. 
Figure 10 and Figure 11 show the slope of the sound power levels (coloured solid lines), calculated with the different models, in function of time. The trend of the two graphs is slightly different, probably because of the different traffic conditions observed in both directions. Similarly, to the previous applications, NMPB is the model that has several fluctuations and, together with ASJ-steady, returns the lower values than the others. Moreover, in this route the fluctuations are evident in all the models, because of the continuous, albeit small, speed variations. Finally, it is also possible to observe the almost total overlap between the graphs of SonRoad and Cnossos, even though the two models are quite different.

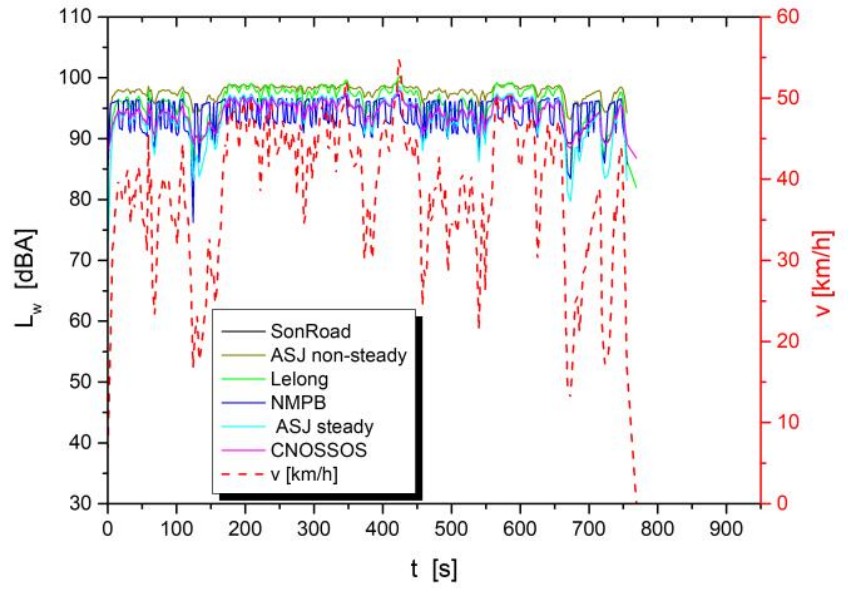

Figure 10. National road route Salerno-Baronissi: Speed profile (in dashed red line, secondary axis) and sound power level $L_{w}$ of the different models (solid lines).

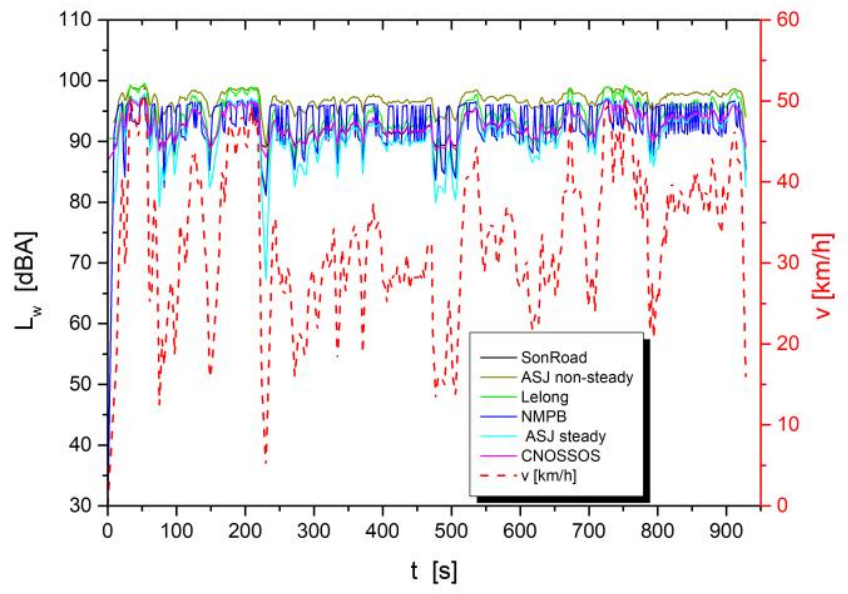

Figure 11. National road route Baronissi-Salerno: Speed profile (in dashed red line, secondary axis) and sound power level $L_{w}$ of the different models (solid lines).

\section{Quantitative comparison}

In order to compare the five models, table 3 shows the results of the calculation of the average and total sound power levels. The aggregated indicators confirm a substantial proximity of the results between the models. In the average line of the table, an important result can be observed. The average of the levels of the different models presents lower values for the urban road of about $5.5 \mathrm{dBA}$ for $\mathrm{L}_{\mathrm{w}, \mathrm{m}}$ and approximately $6.1 \mathrm{dBA}$ for $\mathrm{L}_{\mathrm{w}, \text { tot }}$ than the ones of the highway. If compared with a gain of only about 4 minutes estimated in the travel time, the highway route determines a significant increase of the sound power level emitted by a light vehicle. However, the highway crosses a lower number of residential areas. Therefore, for a more complete assessment of noise impact on the inhabitants of the area, it is necessary to estimate the number of people who actually are affected by the noise levels produced by the two different roads. A possible estimation can be done by density of population per $\mathrm{km}^{2}$, data provided by national authorities, or by social, mobile or wi-fi accesses (see for instance [29]).

Table 3. Comparison of the models' results in the case study. All values are given in dBA.

\begin{tabular}{|c|c|c|c|c|c|c|}
\hline & \multicolumn{4}{|c|}{ Highway } & \multicolumn{2}{|c|}{ National Road } \\
\hline & \multicolumn{2}{|c|}{$\begin{array}{c}\text { Baronissi- } \\
\text { Salerno }\end{array}$} & \multicolumn{2}{|c|}{$\begin{array}{l}\text { Salerno- } \\
\text { Baronissi }\end{array}$} & $\begin{array}{l}\text { Baronissi- } \\
\text { Salerno }\end{array}$ & \multirow{2}{*}{$\begin{array}{l}\text { Salerno- } \\
\text { Baronissi } \\
\mathrm{L}_{\mathrm{w}, \mathrm{m}} \mathrm{L}_{\mathrm{w}, \mathrm{tot}}\end{array}$} \\
\hline & $\mathrm{L}_{\mathrm{w}, \mathrm{m}}$ & $\mathrm{L}_{\mathrm{w}, \text { tot }}$ & $\mathrm{L}_{\mathrm{w}, \mathrm{m}}$ & $\mathrm{L}_{\mathrm{w}, \text { tot }}$ & $\mathrm{L}_{\mathrm{w}, \mathrm{m}} \mathrm{L}_{\mathrm{w}, \text { tot }}$ & \\
\hline Lelong & 102.9 & 129.3 & 102.9 & 129.4 & $95.2 \quad 121.4$ & 97.7122 .3 \\
\hline SonRoad & 102.0 & 128.5 & 101.9 & 128.5 & $\begin{array}{lll}93.6 & 119.8\end{array}$ & 94.9120 .5 \\
\hline NMPB & 98.0 & 124.5 & 97.4 & 123.9 & $\begin{array}{lll}94.3 & 120.5\end{array}$ & 94.8120 .3 \\
\hline $\begin{array}{l}\text { ASJ } \\
\text { non-steady }\end{array}$ & 100.1 & 126.6 & 101.2 & 126.7 & $\begin{array}{lll}97.3 & 123.4\end{array}$ & 97.9123 .5 \\
\hline ASJ steady & 101.6 & 128.1 & 101.6 & 128.1 & $\begin{array}{lll}93.1 & 119.2\end{array}$ & 94.7120 .3 \\
\hline CNOSSOS & 100.9 & 127.4 & 100.9 & 127.5 & $97.3 \quad 123.4$ & 94.7120 .3 \\
\hline Average & 100.9 & 127.4 & 101.0 & 127.4 & 95.1121 .3 & 95.8121 .2 \\
\hline
\end{tabular}

\section{CONCLUSIONS}

In this paper, different sound power emission models have been analysed by comparing the predictive power levels of a single light vehicle in different conditions. The analysis has enabled the evaluation of the trend of the models in different situations, considering several values of speed and acceleration of the vehicle. The definition of the kinematics has involved both simulations and measured data, in urban or highway routes.

The results revealed several trends for the models, as might be expected given the different mathematical structures. In particular, the NMPB model always tended to exhibit a highly variable slope and lower values of emitted noise than the other models. On the contrary, Cnossos seemed to be more stable in the various applications.

A quantitative comparison of the noise emissions estimated by the selected models has been proposed through the definition of two indicators. The first one is the average emitted noise level, defined as the level of the time average of the instantaneous sound power of the vehicle during the trip. The second one is the total emitted noise level, defined summing up all the sound emitted levels during the trip. 
Comparing these two indicators in the proposed applications, it was possible to observe a general flattening of the differences between the selected calculation techniques. In particular, the estimation of these indicators in the application of measured data during two real routes that connect the same origin and destination points, allowed the conclusion that, sometimes, the benefit obtained in terms of travel time involves a significant burden on the noise emitted from the vehicle. This result could affect the people choices for different routes according to their sensibility to the impact of sound emission.

For further developments, the two defined indicators could also be evaluated by connecting the noise data with geographical and population data. For example, population exposure could be reckoned by confronting noise data with land-use rates data. Other possible estimations can be done by density of population per $\mathrm{km}^{2}$, provided by national authorities, or by social, mobile or wi-fi accesses. Thus, an estimation of the inhabitants exposure to vehicle noise levels (i.e. the distribution of noise exposure in the population) could be evaluated by combining the sound levels comprised within a range of values over a territory and its demographic distribution.

In conclusion, the proposed procedure to compare, in terms of noise produced during the trip using Noise Emission Models, more routes connecting the same origin and destination points, showed to be a relevant tool to increase the sensitivity to the issue of the reduction of noise emissions. This aim can be obtained through different actions: the purchase of new and less noisy vehicles, the awareness of adopting a less aggressive driving style and the choice of routes with a less impact of sound emissions.

\section{ACKNOWLEDGMENT}

The Authors are grateful to Prof. Quartieri, for inspiring and supporting this research. The Authors also acknowledge the contribution of Giulia Zotto to the simulation, data collection and analysis, during her bachelor thesis.

\section{REFERENCES}

[1] EEA, "Environmental noise in Europe - 2020", Report no. 22/2019, 2020 [Online]. Available:

https://www.eea.europa.eu/publications/environmentalnoise-in-europe

[2] EC. Directive 2002/49/EC of the European parliament and the Council of 25 June 2002 relating to the assessment and management of environmental noise, Official Journal of the European Communities, 2002, 189, pp. 12-25.

[3] C. Guarnaccia, T. L. L. Lenza, N. E. Mastorakis and J. Quartieri, "A comparison between traffic noise experimental data and predictive models results", Int. J. of Mech., vol. 5, no. 4, pp. 379-386, Jan. 2011.

[4] C. Guarnaccia, J. Bandeira, M. C. Coelho, P. Fernandes, J. Teixeira, G. Ioannidis and J. Quartieri, "Statistical and semi-dynamical road traffic noise models comparison with field measurements", in Proc. 2018 AIP Conf., vol. 1982 (020039), pp.1-6.

[5] C. Guarnaccia, "Advanced tools for traffic noise modelling and prediction", in 2013 WSEAS Trans. Syst., vol. 12, pp. 121-130.

[6] C. Guarnaccia, J. Quartieri, N. E. Mastorakis and C. Tepedino, "Development and application of a time series predictive model to Acoustical noise levels", in 2014 WSEAS Trans. Syst., vol. 13, pp. 745-756.

[7] C. Guarnaccia, J. Quartieri, E. R. Rodrigues and C. Tepedino, "Acoustical noise analysis and prediction by means of multiple seasonality time series model", in Int. J. of Math. Mod. Meth. Appl. Sci., vol. 8, pp. 384-393, Jan. 2014.

[8] C. Steele, "A critical review of some traffic noise prediction models", Appl. Acoust., vol. 62, pp. 271-287, Mar. 2001.

[9] F. Cirianni and G. Leonardi, "Environmental modeling for traffic noise in urban area", American Journal of Environmental Science, vol. 8, no. 4, pp. 345-51, Jun. 2012

[10]A. Can and P. Aumond, "Estimation of road traffic noise emissions: The influence of speed and acceleration", Transportation Res. D-Tr. E., vol. 58, pp. 155-171, Jan. 2018.

[11]C. Guarnaccia, "EAgLE: Equivalent acoustic level estimator proposal", Sensors, vol. 20, no. 3, 701, Jan. 2020.

[12]C. Scrosati, F. Scamoni, G. Zambon. "Uncertainty of façade sound insulation in buildings by a Round Robin Test”, Appl. Acoust., vol. 96, pp. 27-38, Sept. 2015.

[13] G. Iannone, C. Guarnaccia and J. Quartieri, "Noise fundamental diagram deduced by traffic dynamics", in Proc. 2011 WSEAS Int. Conf. EMESEG '11, pp. 501507.

[14] N. Garg and S. Maji, "A critical review of principal traffic noise models: Strategies and implications", Environ. Impact Assess., vol. 46, no. 1, pp. 68-81, Apr. 2014.

[15] J. Quartieri, G. Iannone and C. Guarnaccia, "On the Improvement of Statistical Traffic Noise Prediction Tools", in Proc. 2010 WSEAS Int. Conf. AMTA '10, pp. 201-207.

[16]G. Iannone, C. Guarnaccia and J. Quartieri, "Speed distribution influence in road traffic noise prediction", in Environ. Eng. Manag. J., vol. 12, np.3, pp. 493-501, Mar. 2013.

[17] J. F. Hamet, F. Besnard, S. Doisy, J. Lelong and E. Le Duc "New vehicle noise emission for French traffic noise prediction", in Appl. Acoust., vol. 71, no. 9, pp. 861-869, September 2010.

[18] J. Quartieri, N. E. Mastorakis, G. Iannone, C. Guarnaccia, S. D'Ambrosio, A. Troisi and T. L. L. Lenza, "A review of traffic noise predictive models", in Proc. 2009 WSEAS Int. Conf. MECHANICS'09, pp 72-80.

[19] J. Quartieri, N. E. Mastorakis, G. Iannone, C. Guarnaccia, "Cellular automata application to traffic noise control", in Proc. 2010 WSEAS Int. Conf. ACMOS '10, pp. 299-304.

[20] J. Lelong, "Vehicle noise emission: evaluation of tyre/road and motor-noise contributions", in Proc. of 1999 
Int. Cong. On Noise Control - Internoise 99, vol. 1, pp. 203-08.

[21]K. Heutschi, "SonRoad: new swiss road traffic noise model", in Acta Acust. United Acust., vol. 90, no. 3, pp. 548-554, May 2004.

[22]F. Besnard, J.F. Hamet, J. Lelong, E. Le Duc, V. Guizard, N. Fürst, S. Doisy and G. Dutilleux, "Road noise prediction. 1 - Calculating sound emissions from road traffic. Methodologic Guide", Ed. Sétra, 2009.

[23]F. Besnard, J. Defrance, M. Bérengier, G. Dutilleux, F. Junker, D. Ecotière, E. Le Duc, M. Baulac, B. Bonhomme, J.-P. Deparis, B. Gauvreau, V. Guizard, H. Lefèvre, V. Steimer, D. Van Maercke and V. Zouboff, "Road noise prediction 2 - Noise propagation computation method including meteorological effects (NMPB 2008). Methodologic Guide”, Ed Sétra, 2009.

[24] K. Yamamoto, "Road traffic noise prediction model "ASJ RTN-Model 2008": report of the research committee on road traffic noise", in Acoust. Sci. Technol., vol. 31, no. 1, pp. 2-55, 2010.

[25] S. Kephalopoulos, M. Paviotti, F. Anfosso- Lédée "Common Noise Assessment Methods in Europe (CNOSSOS- EU)", in EUR25379EN, Luxembourg: Publications Office of the European Union, 2012.

[26] R. A. Melo, R. L. Pimentel, D. M. Lacerda and W.M. Silva, "Applicability of models to estimate traffic noise for urban roads", J. Environ. Health Sci. Eng., vol. 13, art. no. 83, Dec. 2015.

[27]E. Hammer, S. Egger, T. Saurer and E. Bühlmann, "Traffic noise emission modelling at lower speed", in Proc. of 2016 Inter. Congr. on Sound \& Vibration ICSV23, pp. 1-9.

[28] Available: http://www.gpxscan.com , last accessed on October 6, 2020

[29] J. M. Bandeira, P. Tafidis, E. Macedo, J. Teixeira, B. Bahmankhah, C. Guarnaccia and M. C. Coelho, "Exploring the Potential of Web Based Information of Business Popularity for Supporting Sustainable Traffic Management", Transp \& Telecommun J., Vol. 21, no. 1, pp. 47-60, Feb. 2020.

\section{Creative Commons Attribution License 4.0 (Attribution 4.0 International, CC BY 4.0)}

This article is published under the terms of the Creative Commons Attribution License 4.0

https://creativecommons.org/licenses/by/4.0/deed.en_US 